\title{
Diurnal and nocturnal feeding rate in Kentish plovers Charadrius alexandrinus on an intertidal flat as recorded by telescopic video systems
}

Tomohiro Kuwae

Published online: 16 January 2007

(C) Springer-Verlag 2007

Erratum to: Mar Biol DOI 10.1007/s00227-006-0506-y

Unfortunately, Table 1 contained an error. The correct table is shown below.

The online version of the original article can be found at http://dx.doi.org/10.1007/s00227-006-0506-y.

T. Kuwae $(\square)$

Coastal and Estuarine Environment Research Group,

Port and Airport Research Institute, 3-1-1, Nagase,

Yokosuka 239-0826, Japan

e-mail: kuwae@ipc.pari.go.jp 
Table 1 Model selection results for foraging attempt rate (FAR) in Kentish plovers

\begin{tabular}{|c|c|c|c|c|c|c|c|c|c|c|c|c|c|}
\hline & Site & Temp & Wind & TAE & Light & $\begin{array}{l}\text { Site } \times \\
\text { Wind }\end{array}$ & $\begin{array}{l}\text { Site } \times \\
\text { Temp }\end{array}$ & $\begin{array}{l}\text { Site } \times \\
\text { Light }\end{array}$ & $\begin{array}{l}\text { Site } \times \\
\text { TAE }\end{array}$ & $\begin{array}{l}\text { Light } \times \\
\text { Temp }\end{array}$ & $\mathrm{AIC}_{\mathrm{C}}$ & $\Delta_{i}$ & $w_{i}$ \\
\hline \multirow[t]{13}{*}{ AIC best } & $\mathrm{x}$ & $\mathrm{x}$ & $\mathrm{x}$ & & & $\mathrm{x}$ & & & & & 62.79 & 0.00 & 0.21 \\
\hline & $\mathrm{x}$ & $\mathrm{x}$ & $\mathrm{x}$ & $\mathrm{x}$ & & $\mathrm{x}$ & & & & & 63.59 & 0.81 & 0.14 \\
\hline & $\mathrm{x}$ & $\mathrm{x}$ & $\mathrm{x}$ & & & $\mathrm{x}$ & $\mathrm{x}$ & & & & 64.38 & 1.59 & 0.09 \\
\hline & $\mathrm{x}$ & $\mathrm{x}$ & $\mathrm{x}$ & & $\mathrm{x}$ & $\mathrm{x}$ & & $\mathrm{x}$ & & & 64.84 & 2.05 & 0.08 \\
\hline & $\mathrm{x}$ & $\mathrm{x}$ & $\mathrm{x}$ & & $\mathrm{x}$ & $\mathrm{x}$ & & & & & 64.86 & 2.07 & 0.07 \\
\hline & $\mathrm{x}$ & $\mathrm{x}$ & $\mathrm{x}$ & $\mathrm{x}$ & & $\mathrm{x}$ & $\mathrm{x}$ & & & & 64.87 & 2.09 & 0.07 \\
\hline & $\mathrm{x}$ & $\mathrm{x}$ & $\mathrm{x}$ & $\mathrm{x}$ & $\mathrm{x}$ & $\mathrm{x}$ & & $\mathrm{x}$ & & & 64.88 & 2.09 & 0.07 \\
\hline & $\mathrm{x}$ & $\mathrm{x}$ & $\mathrm{x}$ & $\mathrm{x}$ & & $\mathrm{x}$ & & & $\mathrm{x}$ & & 65.67 & 2.88 & 0.05 \\
\hline & $\mathrm{x}$ & $\mathrm{x}$ & $\mathrm{x}$ & $\mathrm{x}$ & $\mathrm{x}$ & $\mathrm{x}$ & & & & & 65.69 & 2.90 & 0.05 \\
\hline & $\mathrm{x}$ & $\mathrm{x}$ & $\mathrm{x}$ & $\mathrm{x}$ & $\mathrm{x}$ & $\mathrm{x}$ & $\mathrm{x}$ & $\mathrm{x}$ & & & 65.88 & 3.10 & 0.04 \\
\hline & $\mathrm{x}$ & $\mathrm{x}$ & $\mathrm{x}$ & & $\mathrm{x}$ & $\mathrm{x}$ & $\mathrm{x}$ & $\mathrm{x}$ & & & 66.15 & 3.36 & 0.04 \\
\hline & $\mathrm{x}$ & $\mathrm{x}$ & $\mathrm{x}$ & $\mathrm{x}$ & $\mathrm{x}$ & $\mathrm{x}$ & & $\mathrm{x}$ & & $\mathrm{x}$ & 66.24 & 3.46 & 0.04 \\
\hline & $\mathrm{x}$ & $\mathrm{x}$ & $\mathrm{x}$ & & $\mathrm{x}$ & $\mathrm{x}$ & $\mathrm{x}$ & & & & 66.37 & 3.59 & 0.04 \\
\hline Selection probability & 1.00 & 1.00 & 1.00 & 0.47 & 0.43 & 1.00 & 0.29 & 0.27 & 0.05 & 0.04 & & & \\
\hline $\bar{\beta}$ & 0.610 & 0.019 & 0.021 & -0.005 & 0.016 & -0.100 & 0.006 & -0.054 & 0.000 & -0.001 & & & \\
\hline $\mathrm{SE}(\bar{\beta})$ & 0.133 & 0.008 & 0.014 & 0.007 & 0.041 & 0.034 & 0.012 & 0.091 & 0.001 & 0.002 & & & \\
\hline Bias & 0.2 & 0.4 & 0.7 & 1.5 & 2.7 & 0.3 & 2.1 & 1.7 & 4.0 & 2.4 & & & \\
\hline
\end{tabular}

The predictor variable included in the selected models is shown as $\mathrm{x}$

$T A E$ time after emersion; $\Delta_{i}$ AIC difference calculated as $\mathrm{AIC}_{\mathrm{i}}-\mathrm{AIC}$ best; $w_{i}$ Akaike weight showing the probability that model $i$ would be selected as the best fitting model in the confidence set; $\bar{\beta}$ model-averaged coefficient estimate; $\bar{\beta}$ for categorical predictor variables (site and light) indicates a difference between two intercepts, i.e. Site A - Site B for site and night - day for light; $\bar{\beta}$ for interactions indicates a difference between two slopes, for example, slope for wind speed at Site B is 0.021 (appeared in $\bar{\beta}$ for wind) and that at Site $\mathrm{A}$ is -0.100 (appeared in $\bar{\beta}$ for site $\times$ wind) $+0.021 ; \mathrm{SE}(\bar{\beta})$ standard error of $\bar{\beta} ;$ Bias bias on coefficient estimate calculated as $\mathrm{SE}(\bar{\beta}) /|\bar{\beta}|$ 